\title{
Experimental and theoretical approach on the optical properties of zinc borotellurite glass doped with dysprosium oxide
}

\begin{abstract}
A series of glass samples with chemical formula $\{[(\mathrm{TeO} 2) 0.7(\mathrm{~B} 2 \mathrm{O} 3) 0.3] 0.7(\mathrm{ZnO}) 0.3\} 1-\mathrm{x}(\mathrm{Dy} 2 \mathrm{O} 3) \mathrm{x}$ where $\mathrm{x}=0.01,0.02,0.03,0.04$ and $0.05 \mathrm{M}$ fraction were synthesized through conventional melt-quenching method. The most common way to fabricate a glass material is by fusion of two or more component oxides followed by their quenching. This technique is known as melt-quenching technique. Kaur et al. (2016) [1] highlighted that the melt-quenching method able to enhance the mechanical properties like hardness and flexural strength of the material. The nature of the glass systems is proven to be amorphous based on the XRD pattern. The FTIR spectra of the glass systems confirm the existence of five bands which are assigned for the $\mathrm{BO} 4, \mathrm{BO} 3, \mathrm{TeO} 4$ and $\mathrm{TeO} 3$ vibrational groups. The density of the glass systems is increased with the addition of Dy2O3 while the molar volume is found to be inversely proportional to the density of the proposed glass. The optical properties of the glasses are determined through the absorption spectra obtained from the UV-VIS spectrophotometer. From the absorption spectra, the indirect and direct optical band gaps and the Urbach energy are found to be inversely proportional to each other. As the molar fraction of the Dy2O3 increased, the optical band gaps are observed to increase as opposed to the Urbach energy. For this glass system, the values of refractive index, electronic polarizability, oxide ion polarizability and the optical basicity are found to decrease as the addition of the dysprosium oxide is increased. From the emission spectra, two intense blue and yellow emission bands are observed, which correspond to the $4 \mathrm{~F} 9 / 2 \rightarrow 6 \mathrm{H} 15 / 2$ and $4 \mathrm{~F} 9 / 2 \rightarrow 6 \mathrm{H} 13 / 2$ transitions of Dy $3+$ ions respectively. The CIE chromaticity coordinates of the zinc borotellurite glass systems are found to be located in the white light region.
\end{abstract}

Keyword: Dysprosium oxide; Electronic polarizability; Emission analysis; Optical bad gap; Optical basicity; Oxide ion polarizability; Refractive index; Zinc borotellurite glasses 